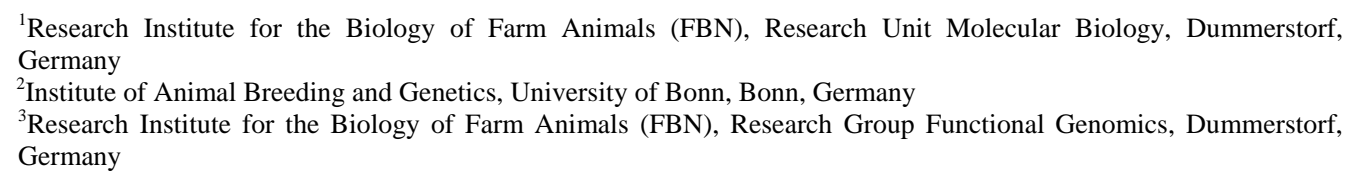

KLAUS WIMMERS ${ }^{1}$, NARES TRAKOOLJUL ${ }^{2}$, KARL SCHELLANDER ${ }^{2}$, and SIRILUCK PONSUKSILI ${ }^{3}$

\title{
Polymorphisms of the androgen receptor gene associate with fatness, uterus and ovary measurements in the pig
}

Dedicated to Prof. Dr. Dr. h.c. mult. Ernst Kalm on the occasion of his $65^{\text {th }}$ birthday

\begin{abstract}
Quantitative trait loci, QTL, for fatness and carcass traits in pigs have been recently mapped to the $\mathrm{X}$ chromosome approximately at the position where the androgen receptor gene (AR) is localized. The AR acts as a nuclear transcription factor regulating expression of a number of androgenic response genes during various stages of sexual development. The present study aimed to analyze association of the $A R$ genotype on traits related to fatness and phenotype of primary female sexual organs, ovary and uterus. Animals of a F2 resource population based on Duroc and Berlin Miniature pig were genotyped at a multi-allelic microsatellite marker (CCTTT)n situated in the $5^{\prime}$ untranslated region and a bi-allelic CAG-insertion/deletion polymorphism (CAGINDEL) within exon 1 of the $A R$. Association analysis showed that the $A R$ genotype both at the CAG-INDEL and microsatellite affect almost all fatness traits measured. The D allele inherited from Duroc was associated with the decrease of fat thickness. The $A R$ genotypes also affect uterus and ovary measurements. The pigs homozygous for D allele were likely to have a lighter uterus, shorter uterus horns, oviducts and smaller ovaries than pigs homozygous for the M allele. Our results confirm the previously reported QTL for fatness traits and provide evidence for a QTL affecting dimensions of uterus and ovary on the X chromosome. The AR is a positional functional candidate gene for both trait complexes.
\end{abstract}

Key Words: sexual hormone, obesity, carcass, lean meat, reproductive organ, fertility, QTL

\section{Zusammenfassung}

Titel der Arbeit: Polymorphismen im Androgenrezeptorgen sind assoziiert mit Speck- und Uterus- und Ovarmaßen beim Schwein

Genorte für quantitative Merkmale, quantitative trait loci, QTL, für Speckmaße und Schlachtkörpermerkmale beim Schwein sind auf dem X-Chromosom nahe dem Androgenrezeptorgen (AR) kartiert worden. Der AR ist ein Transkriptionsfaktor, der die Expression einer Reihe von Androgen-abhängigen Genen während verschiedener Stadien der sexuellen Entwicklung reguliert. Die vorliegende Untersuchung zielt auf Zusammenhänge zwischen AR-Genotyp und Merkmalen der Körperverfettung und der Ausformung primärer weiblicher Geschlechtsorgane, Uterus, Ovar. Tiere einer F2 Ressourcenpopulation, basierend auf Duroc und Berliner Miniaturschwein, wurden an einem multi-allelischen Mikrosatellitenmarker (CCTTT)n im 5' untranslatierten Bereich sowie einer bi-allelischen CAG-insertion/deletion (CAG-INDEL) im Exon 1 des AR genotypisiert. Die Assoziationsanalyse zeigte, dass der AR-Genotyp am CAG-INDEL und am Mikrosatellit mit fast allen erhobenen Speckmaßen im Zusammenhang steht. Das D-Allel, das vom Duroc vererbt wurde, war mit der Abnahme der Speckmaße verbunden. Die AR Genotypen beeinflussen auch Gebärmutter- und Eierstockmaße. Die Schweine, die für D-Allel homozygot sind, weisen kleinere Uteri, kürzere Uterushörner, Eileiter und kleinere Eierstöcke auf als die Schweine, die für das M-Allel homozygot sind. Die Ergebnisse bestätigen zuvor berichtete QTL für Speckmaße und liefern Evidenz für die Existenz eines QTL für Uterus- und Ovarmaße auf dem X-Chromosom. Das AR ist ein positionelles, funktionelles Kandidatengen für beide Merkmalskomplexe. 


\section{Introduction}

The androgen receptor is classically activated by the binding of androgens. Two most important androgens are testosterone and 5 $\alpha$-dihydrotestosterone. After liganddependent activation the hormone-receptor complexes bind to hormone response elements (HRE) of target genes and interact with other basal transcription factors, coactivators and co-repressors to up or down regulate transcription of the target genes. A number of genes participating in cell metabolism, activity and response have been shown to be regulated by androgens (NANTERMET et al., 2004; NELSON et al., 2002). The $A R$ has been shown to be expressed in diverse tissues like prostate, testis, ovary, uterus, mammary gland, anterior pituitary, thyroid, adrenal cortex, liver, muscle as well as bone during stages suggesting its importance for normal development and function of those tissues (PELLETIER, 2000; SLOMCZYNSKA et al., 2001; TRIPEPI et al., 2000). Mutations of the $A R$ associate with various human disorders like male infertility, spinal and bulbar muscular atrophy (SBMA), breast, and prostate cancers (RIS-STALPERS et al., 1990; McPHAUL et al., 1993; BRINKMANN, 2001). The $A R$ effects on development of reproductive organs/tissues like follicle and testis have been reported in the pig (SLOMCZYNSKA et al., 2001; TRIPEPI et al., 2000). Quantitative trait loci, QTL, for various traits like back fat, abdominal fat, intramuscular fat content, and carcass traits were mapped to the porcine $X$ chromosome in close vicinity to AR (KNOTT et al., 1998; ROHRER and KEELE, 1998; HARLIZIUS et al., 2000; ROHRER, 2000; MALEK et al., 2001a,b). Hence, the $A R$ is a functional and positional candidate gene for production and reproduction traits in pigs.

Previously, polymorphisms have been reported in coding and promoter regions of the porcine $A R$ gene (TRAKOOLJUL et al., 2004). Moreover, an association between the $A R$ genotype and its mRNA transcript level has been observed. In the present study, the $A R$ genotype at the CAG-insertion/deletion (INDEL) in the exon 1 and the CCTTT(n) microsatellite in 5'-untranslated region were analyzed for associations on production and reproduction traits like fat thickness, uterus and ovary dimensions.

\section{Materials and methods}

Animals and phenotype records

Analyses were done in a three-generation porcine F2 resource population (DUMI resource population) that was established by reciprocal crossbreeding of Duroc and Berlin Miniature Pig breeds (HARDGE et al., 1999). F2 piglets were weaned at about 6 weeks of age and kept in flat decks until day 100 and subsequently in single pens until slaughter at 200 days of age. The following traits related to fatness were recorded: average back fat, fat depth at shoulder, fat depth at $10^{\text {th }} \mathrm{rib}$, loin fat depth, and fat depth at side. Dimensions of reproductive organs, vagina, cervix, uterus and ovary were recorded at slaughter. All sows were observed for estrous cycle (1) or non estrous (0).

\section{Genotype analysis}

Genomic DNA was isolated from tail cuts by standard procedure of proteinase K digestion and phenol-chloroform extraction. Genotyping of the CCTTT(n) microsatellite at 5'-UTR of the pig $A R$ was performed as previously described 
(TRAKOOLJUL et al., 2000). The CAG-INDEL genotyping was based on PCR amplification length polymorphism using primers: forward 5'-agctgctccaccgatcttaaag$3^{\prime}$ and reward 5'-cttacacaattccttggcgctg-3'. The PCR was prepared in a total volume of $10 \mu \mathrm{l}$ containing $1 \mathrm{x}$ PCR buffer, $0.25 \mathrm{mM}$ dNTPs, $0.25 \mu \mathrm{M}$ of each primer, $0.25 \mathrm{U}$ of Taq polymerase, and $25 \mathrm{ng}$ of DNA. Touch-down step PCR was performed in Thermocycler (MJ research) programmed at $94{ }^{\circ} \mathrm{C}$ for $3 \mathrm{~min}, 10 \mathrm{x}\left[94^{\circ} \mathrm{C} 30 \mathrm{sec}, 63-\right.$ $58{ }^{\circ} \mathrm{C}\left(-0.5^{\circ} \mathrm{C} / \mathrm{cycle}\right) 30 \mathrm{sec}, 72^{\circ} \mathrm{C} 1 \mathrm{~min}$ ], 24 x [94 ${ }^{\circ} \mathrm{C} 30 \mathrm{sec}, 58{ }^{\circ} \mathrm{C} 30 \mathrm{sec}, 72{ }^{\circ} \mathrm{C} 1$ min] with additional extension of $72{ }^{\circ} \mathrm{C}$ for $5 \mathrm{~min}$. Amplified fragments were separated on $8 \%$ denatured polyacrylamide gel, run in 1 x TBE buffer at 50 watts for 90 min using vertical electrophoresis system. Mendelian inconsistency of genotype in the pedigree was eliminated using PedCheck program ( $\mathrm{O}^{\prime}$ CONNELL and WEEKS 1998).

\section{Association analysis}

Statistic analyses were examined with SAS for Windows version 8.2, using the procedure PROC MIXED. Association effects of the $A R$ (CAG-INDEL) and microsatellite (CCTTTn) genotypes on fatness traits were analyzed with the statistical model (1) including fixed effects of parity and genotype and random effects of family (a combination of F1 boar x F1 sow), separately for the sexes. Individual slaughter weight was in the model 1 as covariate. For uterus measurements, gilt status ( $0=$ noncyclic or 1 = cyclic) and genotype were included as fixed effects where as family as random effects in the model (2). Results are presented as least square means (LSM) and standard errors of least square means (SE). All multiple comparisons were performed with adjustments according to Tukey-Kramer. Statistical models used for the analyses are as follows:

Model (1) for fatness traits:

$y_{i j k l}=\mu+$ parity $_{i}+$ genotype $_{j}+$ family $_{k}+b$ (Slaughter weight $)_{i j k l}+e_{i j k l}$

where:

parity (fixed effect)

genotype (fixed effect)

or

slaughter weight family (random effect)

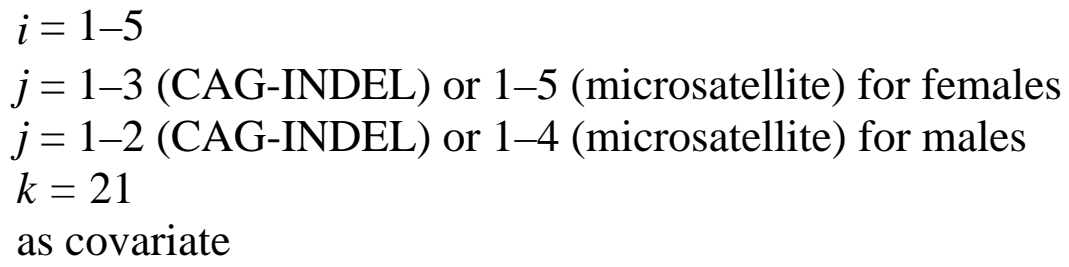

Model (2) for uterus and ovary measurements:

$y_{i j k l}=\mu+$ gilt status $_{i}+$ genotype $_{j}+$ family $_{k}+e_{i j k l}$

where:

gilt status (fixed effect) $\quad i=1-2$

genotype (fixed effect) $\quad j=1-3$ (CAG-INDEL) or 1-5 (microsatellite)

family (random effect) $\quad k=1-26$

\section{Results}

Allele segregation

Genotyping of the DUMI animals for the CAG-INDEL showed two segregating alleles with allele size of 213 and 216 bp, namely " $M$ " and " $D$ " allele, respectively. This 
CAG repeat codes for polyglutamine, which is corresponding to amino acid position 179 to 188 of the pig $A R$. The D allele predicts for 10 glutamine residues and is a common allele found in Duroc and other breeds. The $\mathrm{M}$ allele codes for 9 glutamine residues and is an alternative deleted allele detected in Berlin Miniature pig. At the CCTTT(n) microsatellite, four segregating alleles with 188, 203, 204 and 228 bp were observed (Figure 1). Sequencing and genotyping of the DUMI founders revealed four haplotypic combinations: M-204, M-228, D-188, and D-203. Since the Duroc and Berlin Miniature pig founders were fixed for different alleles at the CAG-INDEL, all F1 sows were heterozygous and the genotypic ratio of their F2 followed X-linked inheritance pattern.

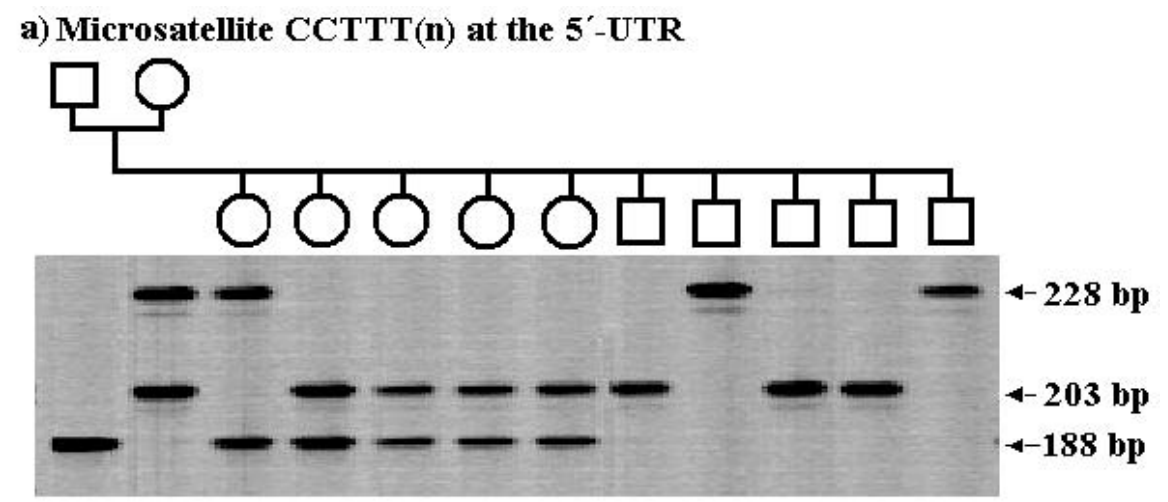

b) The CAG-INDEL in exon 1

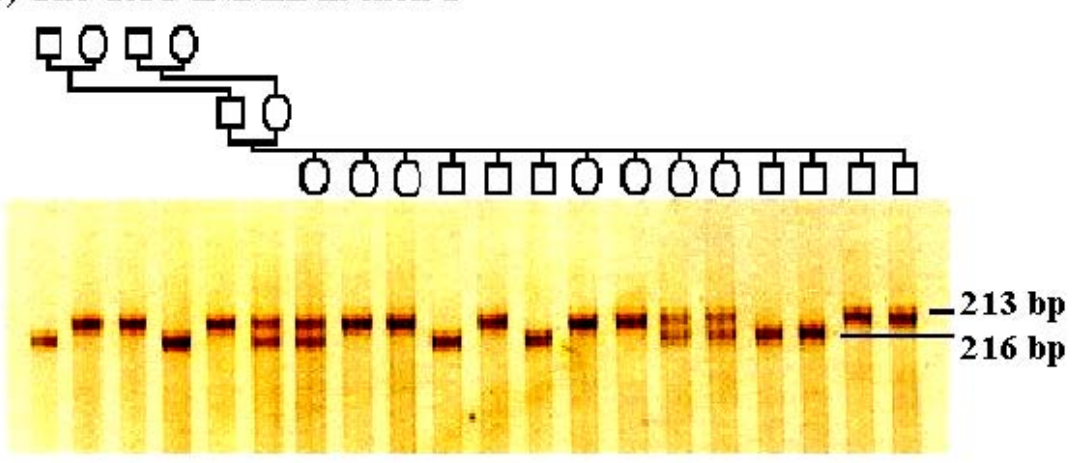

Fig. 1: X-linked inheritance of the microsatellite CCTTT(n) (a) and the CAG-INDEL (b) in DUMI F2 families (X-gekoppelte Vererbung des Mikrosatelliten CCTTT(n) (a) und der CAG-INDEL (b) in DUMI F2-Familien)

Association analysis - fatness traits

Association analyses for fatness traits are shown in Table 1 . The results showed that the $A R$ genotype at the CAG-INDEL was associated with all fatness traits measured. However, significant differences of LSM were found in males but not in females. The $\mathrm{D}$ allele from Duroc was associated with low fatness while the $\mathrm{M}$ allele from the Berlin miniature pig with high fatness. Average back fat thickness of male pigs carrying the $\mathrm{M}$ allele was $3.22 \mathrm{~mm}$ higher than of pigs with $\mathrm{D}$ allele.

No significant effect was found between the microsatellite genotype and fatness traits in females. However, pigs with the 188/188 and 188/203 genotypes, i.e. with both alleles from Duroc, tend to deposit less fat than other genotypes. Pigs with genotype 
with both alleles from Berlin Miniature pig were dropped from comparisons because of small number of animals available. Allele effects on fatness were more pronounced in males than in females. Male pigs with genotype 204/0 were significantly different from pigs with genotype $203 / 0$ or $188 / 0$ in all fat traits except fat depth at side as shown in Table 1.

Table 1

Effects of the $A R$ genotypes on fatness traits (LSM $\pm \mathrm{SE}$ ) (Effekte der AR Genotypen auf Speckmaße)

\begin{tabular}{|c|c|c|c|c|c|c|c|}
\hline \multirow[b]{2}{*}{ Sex } & \multirow[b]{2}{*}{ Genotype } & \multirow[b]{2}{*}{$\begin{array}{l}\text { Origin of } \\
\text { alleles }\end{array}$} & \multicolumn{5}{|c|}{ Fatness traits $[\mathrm{cm}]$} \\
\hline & & & $\begin{array}{l}\text { Average } \\
\text { back fat }\end{array}$ & $\begin{array}{l}\text { Shoulder } \\
\text { fat depth }\end{array}$ & $\begin{array}{l}\text { Fat depth } \\
\text { at side }\end{array}$ & $\begin{array}{l}\text { Fat depth } \\
\text { at } 10^{\text {th }} \mathrm{rib}\end{array}$ & $\begin{array}{c}\text { Loin } \\
\text { fat depth }\end{array}$ \\
\hline \multirow{7}{*}{ q } & $\mathrm{M} / \mathrm{M}$ & & 4.326 & 5.634 & 5.941 & 3.526 & 3.819 \\
\hline & $(n=17)$ & & $\pm 0.182^{\mathrm{a}, \mathrm{b}}$ & $\pm 0.236^{\mathrm{a}, \mathrm{b}}$ & $\pm 0.282^{\mathrm{a}, \mathrm{b}}$ & \pm 0.179 & \pm 0.194 \\
\hline & $\mathrm{D} / \mathrm{M}$ & & 4.255 & 5.532 & 5.752 & 3.472 & 3.757 \\
\hline & $(n=100)$ & & $\pm 0.091^{b}$ & $\pm 0.110^{b}$ & $\pm 0.131^{\mathrm{b}}$ & \pm 0.090 & \pm 0.098 \\
\hline & $\mathrm{D} / \mathrm{n}(\mathrm{n}=92)$ & & 4.016 & 5.214 & 5.339 & 3.301 & 3.534 \\
\hline & $D / D(11-92)$ & & $\pm 0.093^{\mathrm{a}}$ & $\pm 0.112^{\mathrm{a}}$ & $\pm 0.134^{\mathrm{a}}$ & \pm 0.092 & \pm 0.100 \\
\hline & $P$-value & & 0.039 & 0.039 & 0.022 & ns & ns \\
\hline \multirow{4}{*}{0} & $\mathrm{M} / 0(\mathrm{n}=95)$ & & $\begin{array}{r}4.980 \\
+0.085^{a}\end{array}$ & $\begin{array}{r}6.415 \\
+0107^{a}\end{array}$ & $\begin{array}{r}7.100 \\
+0131 \text { a }\end{array}$ & $\begin{array}{r}4.133 \\
+0083^{a}\end{array}$ & 4.393 \\
\hline & & & 4.658 & 6.058 & 6.750 & 3.826 & 4.091 \\
\hline & $\mathrm{D} / 0(\mathrm{n}=90)$ & & $\pm 0.085^{\mathrm{b}}$ & $\pm 0.107^{b}$ & $\pm 0.131^{b}$ & $\pm 0.083^{b}$ & $\pm 0.095^{b}$ \\
\hline & $P$-value & & 0.001 & 0.005 & 0.013 & 0.002 & 0.005 \\
\hline \multirow{11}{*}{ 우 } & 188/188 & $\mathrm{D} / \mathrm{D}$ & 4.123 & 5.305 & 5.526 & 3.461 & 3.593 \\
\hline & $(n=26)$ & D/D & \pm 0.164 & \pm 0.207 & \pm 0.244 & \pm 0.161 & \pm 0.174 \\
\hline & $188 / 228$ & $\mathrm{D} / \mathrm{M}$ & 4.585 & 5.981 & 5.889 & 3.747 & 3.976 \\
\hline & $(n=12)$ & $\mathrm{D} / \mathrm{M}$ & \pm 0.224 & \pm 0.291 & \pm 0.347 & \pm 0.219 & \pm 0.239 \\
\hline & $203 / 204$ & $\mathrm{D} / \mathrm{M}$ & 3.956 & 5.312 & 5.579 & 3.251 & 3.327 \\
\hline & $(n=20)$ & D/MI & \pm 0.223 & \pm 0.273 & \pm 0.318 & \pm 0.222 & \pm 0.235 \\
\hline & $188 / 204$ & & 4.242 & 5.475 & 5.737 & 3.446 & 3.807 \\
\hline & $(n=68)$ & D/M & \pm 0.114 & \pm 0.140 & \pm 0.163 & \pm 0.113 & \pm 0.120 \\
\hline & $188 / 203$ & $\mathrm{D} / \mathrm{R}$ & 3.984 & 5.171 & 5.249 & 3.241 & 3.534 \\
\hline & $(n=66)$ & D/D & \pm 0.112 & \pm 0.138 & \pm 0.161 & \pm 0.111 & \pm 0.118 \\
\hline & $P$-value & & ns & ns & ns & ns & ns \\
\hline \multirow{9}{*}{ 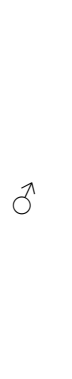 } & $238(n-15)$ & $M$ & 4.638 & 6.003 & 7.078 & 3.956 & 3.965 \\
\hline & $228(11-15)$ & IMI & $\pm 0.187^{\mathrm{a}, \mathrm{b}, \mathrm{c}}$ & $\pm 0.23^{\mathrm{a}, \mathrm{b}, \mathrm{c}}$ & \pm 0.275 & $\pm 0.188^{\mathrm{a}, \mathrm{b}, \mathrm{c}}$ & $\pm 0.203^{\mathrm{a}, \mathrm{b}, \mathrm{c}}$ \\
\hline & $204(\mathrm{n}=80)$ & $M$ & 5.067 & 6.517 & 7.109 & 4.179 & 4.502 \\
\hline & $204(n=80)$ & IMI & $\pm 0.095^{\mathrm{a}}$ & $\pm 0.11^{\mathrm{a}, \mathrm{d}}$ & \pm 0.144 & $\pm 0.095^{\mathrm{a}, \mathrm{d}}$ & $\pm 0.105^{\mathrm{a}}$ \\
\hline & $203(n=64)$ & $\mathrm{D}$ & 4.718 & 6.150 & 6.828 & 3.844 & 4.164 \\
\hline & $203(n=64)$ & D & $\pm 0.099^{b}$ & $\pm 0.124^{\mathrm{b}, \mathrm{d}, \mathrm{e}}$ & \pm 0.149 & $\pm 0.099^{\mathrm{b}, \mathrm{e}}$ & $\pm 0.109^{\mathrm{b}, \mathrm{d}}$ \\
\hline & $188(n=26)$ & $\mathrm{D}$ & 4.506 & 5.818 & 6.532 & 3.783 & 3.911 \\
\hline & & & $\pm 0.160^{\mathrm{b}, \mathrm{c}}$ & $\pm 0.200^{\mathrm{c}, \mathrm{e}}$ & \pm 0.238 & $\pm 0.160^{\mathrm{c}, \mathrm{d}, \mathrm{e}}$ & $\pm 0.175^{\mathrm{c}, \mathrm{d}}$ \\
\hline & $P$-value & & $<0.010$ & $<0.007$ & ns & $<0.027$ & $<0.039$ \\
\hline
\end{tabular}

Association analysis - uterus and ovary measurements

Association analyses showed both the CAG-INDEL and microsatellite genotypes affect uterus measurements. The homozygous DD pigs had significantly higher weight of vagina and cervix than homozygous MM pigs $(p<0.001)$. However, the pigs homozygous for MM genotype were likely to have a heavier uterus and longer uterus horns than homozygous DD pigs. Homozygous MM pigs had longer oviducts and larger ovaries (left and right) than homozygous DD pigs (Table 2). 
Table 2

Effects of the CAG-INDEL genotype of AR on uterus and ovary measures (LSM \pm SE) (Effekte der CAGINDEL Genotypen am AR auf Uterus- und Ovarmaße)

\begin{tabular}{|c|c|c|c|c|}
\hline \multirow[b]{2}{*}{ Trait } & \multicolumn{3}{|c|}{ Genotype } & \multirow[t]{2}{*}{$P$-value } \\
\hline & $\mathrm{D} / \mathrm{D}$ & $\mathrm{D} / \mathrm{M}$ & $\mathrm{M} / \mathrm{M}$ & \\
\hline Vagina and cervix length (cm) & $\begin{array}{c}18.07 \\
\pm 0.56\end{array}$ & $\begin{array}{c}17.85 \\
\pm 0.34\end{array}$ & $\begin{array}{c}17.76 \\
\pm 0.43\end{array}$ & ns \\
\hline Vagina and cervix weight (g) & $\begin{array}{c}50.26 \\
\pm 3.35^{\mathrm{a}}\end{array}$ & $\begin{array}{c}43.59 \\
\pm 2.20^{\mathrm{a}}\end{array}$ & $\begin{array}{c}34.78 \\
\pm 2.79^{b}\end{array}$ & 0.001 \\
\hline Uterine length (cm) & $\begin{array}{c}3.64 \\
\pm 0.39\end{array}$ & $\begin{array}{c}4.40 \\
\pm 0.26\end{array}$ & $\begin{array}{c}4.62 \\
\pm 0.33\end{array}$ & ns \\
\hline Uterine weight (g) & $\begin{array}{c}8.00 \\
\pm 1.51\end{array}$ & $\begin{array}{l}10.04 \\
\pm 1.04\end{array}$ & $\begin{array}{l}10.62 \\
\pm 1.31\end{array}$ & ns \\
\hline Uterine horn length (cm) & $\begin{array}{c}135.62 \\
\pm 10.23\end{array}$ & $\begin{array}{l}148.37 \\
\pm 6.71\end{array}$ & $\begin{array}{l}148.98 \\
\pm 8.43\end{array}$ & ns \\
\hline Uterus horn weight (g) & $\begin{array}{r}163.15 \\
\pm 19.28\end{array}$ & $\begin{array}{l}186.19 \\
\pm 13.13\end{array}$ & $\begin{array}{c}166.47 \\
\pm 16.50\end{array}$ & ns \\
\hline Oviduct length (cm) & $\begin{array}{r}31.85 \\
\pm 1.19^{\mathrm{a}}\end{array}$ & $\begin{array}{c}35.64 \\
\pm 0.76^{\mathbf{b}}\end{array}$ & $\begin{array}{c}38.14 \\
\pm 0.95^{b}\end{array}$ & 0.01 \\
\hline Oviduct weight (g) & $\begin{array}{c}2.66 \\
\pm 0.21\end{array}$ & $\begin{array}{c}2.86 \\
\pm 0.13\end{array}$ & $\begin{array}{c}2.75 \\
\pm 0.16\end{array}$ & ns \\
\hline Ovary length, right (cm) & $\begin{array}{c}2.13 \\
\pm 0.10^{\mathrm{a}}\end{array}$ & $\begin{array}{c}2.35 \\
\pm 0.06^{\text {a,b }}\end{array}$ & $\begin{array}{c}2.54 \\
\pm 0.08^{b}\end{array}$ & 0.007 \\
\hline Ovary length, left (cm) & $\begin{array}{c}2.38 \\
\pm 0.11\end{array}$ & $\begin{array}{c}2.46 \\
\pm 0.08\end{array}$ & $\begin{array}{c}2.47 \\
\pm 0.10\end{array}$ & ns \\
\hline Ovary weight, right (g) & $\begin{array}{c}2.48 \\
\pm 0.43^{\mathrm{a}}\end{array}$ & $\begin{array}{c}3.25 \\
\pm 0.28^{\mathbf{a}, \mathbf{b}}\end{array}$ & $\begin{array}{c}4.09 \\
\pm 0.36^{\mathbf{b}}\end{array}$ & 0.01 \\
\hline Ovary weight, left (g) & $\begin{array}{c}2.83 \\
\pm 0.51^{\mathrm{a}}\end{array}$ & $\begin{array}{c}3.64 \\
\pm 0.31^{\text {a,b }}\end{array}$ & $\begin{array}{c}4.52 \\
\pm 0.38^{b}\end{array}$ & 0.03 \\
\hline Ovary width, right (cm) & $\begin{array}{c}1.43 \\
\pm 0.09\end{array}$ & $\begin{array}{c}1.59 \\
\pm 0.06\end{array}$ & $\begin{array}{c}1.68 \\
\pm 0.08\end{array}$ & ns \\
\hline Ovary width, left (cm) & $\begin{array}{c}1.53 \\
\pm 0.09\end{array}$ & $\begin{array}{c}1.68 \\
\pm 0.06\end{array}$ & $\begin{array}{c}1.77 \\
\pm 0.08\end{array}$ & ns \\
\hline
\end{tabular}

$\overline{\text { a,b }}$ indicate significant differences, $\mathrm{ns}=$ not significant differences, $P$-value is adjusted according to Tukey-Kramer

Similarly, associations between the microsatellite and uterus and ovary measurements were found significant. The pigs carrying genotype 204/228 with both alleles inherited from Berlin Miniature pig were likely to have a heavier uterus body, longer uterus horns and larger ovaries than pigs with the genotype 188/188 with both alleles from Duroc. Moreover, pigs with the genotype 203/228 and 203/204 showed larger uterine horns compared to pigs with the genotype 188/188 (Table 3). 
Table 3

Effects of microsatellite genotype of AR on uterus and ovary measures (LSM \pm SE) (Effekte der Mikrosatelliten Genotypen am AR auf Uterus- und Ovarmaße)

\begin{tabular}{|c|c|c|c|c|c|c|}
\hline \multirow[b]{2}{*}{ Trait } & \multicolumn{5}{|c|}{ Microsatellite genotype (allele inheritance) } & \multirow[t]{2}{*}{$P$-value } \\
\hline & $\begin{array}{c}204 / 228 \\
(\mathrm{M} / \mathrm{M}) \\
n=53\end{array}$ & $\begin{array}{c}203 / 228 \\
(\mathrm{D} / \mathrm{M}) \\
n=14\end{array}$ & $\begin{array}{c}203 / 204 \\
(\mathrm{D} / \mathrm{M}) \\
n=28\end{array}$ & $\begin{array}{c}188 / 204 \\
(D / M) \\
n=33\end{array}$ & $\begin{array}{c}188 / 188 \\
(D / D) \\
n=31\end{array}$ & \\
\hline Vagina and cervix & 17.86 & 18.68 & 18.21 & 17.22 & 17.94 & \\
\hline length $(\mathrm{cm})$ & \pm 0.43 & \pm 0.89 & \pm 0.57 & \pm 0.56 & \pm 0.56 & ns \\
\hline $\begin{array}{l}\text { Vagina and cervix } \\
\text { weight (g) }\end{array}$ & $\begin{aligned} & 35.16 \\
\pm & 2.94^{\mathrm{a}, \mathrm{b}}\end{aligned}$ & $\begin{array}{c}40.18 \\
\pm 5.47^{\text {a,c,d,e }}\end{array}$ & $\begin{aligned} & 47.01 \\
\pm & 3.70^{\mathrm{c}, \mathrm{f}, \mathrm{g}}\end{aligned}$ & $\begin{array}{c}42.03 \\
\pm 3.41^{\text {b,d,f,h }}\end{array}$ & $\begin{aligned} & 49.79 \\
\pm & 3.46^{\mathrm{e}, \mathrm{g}, \mathrm{h}}\end{aligned}$ & $0.03-0.02$ \\
\hline Uterus length $(\mathrm{cm})$ & $\begin{array}{r}4.78 \\
\pm 0.35\end{array}$ & $\begin{array}{c}4.14 \\
\pm 0.63\end{array}$ & $\begin{array}{c}5.10 \\
\pm 0.43\end{array}$ & $\begin{array}{c}3.94 \\
\pm 0.39\end{array}$ & $\begin{array}{c}3.49 \\
\pm 0.40\end{array}$ & ns \\
\hline Uterus weight (g) & $\begin{array}{c}11.89 \\
\pm 1.36^{\mathrm{a}, \mathrm{b}, \mathrm{c}, \mathrm{d}}\end{array}$ & $\begin{array}{c}9.72 \\
\pm 2.3^{\mathrm{a}, \mathrm{e}, \mathrm{f}, \mathrm{g}}\end{array}$ & $\begin{aligned} & 14.06 \\
\pm & 1.66\end{aligned}$ & $\begin{aligned} & 7.18 \\
\pm & 1.50^{\mathrm{c}, \mathrm{f}, \mathrm{h}}\end{aligned}$ & $\begin{array}{c}6.83 \\
\pm 1.52^{\mathrm{d}, \mathrm{g}, \mathrm{h}}\end{array}$ & $0.02-0.01$ \\
\hline $\begin{array}{l}\text { Uterine horns length } \\
(\mathrm{cm})\end{array}$ & $\begin{array}{r}157.53 \\
\pm 8.21^{\mathrm{a}, \mathrm{b}, \mathrm{c}}\end{array}$ & $\begin{aligned} & 173.21 \\
\pm & 15.77^{\mathrm{a}, \mathrm{d}, \mathrm{e}}\end{aligned}$ & $\begin{aligned} & 172.21 \\
\pm & 10.49^{\mathrm{b}, \mathrm{d}}\end{aligned}$ & $\begin{array}{l}119.24 \\
\pm 9.92^{f}\end{array}$ & $\begin{array}{c}127.30 \\
\pm 9.94^{\text {ce, }, \mathrm{f}}\end{array}$ & $\begin{array}{l}0.04- \\
0.005\end{array}$ \\
\hline $\begin{array}{l}\text { Uterine horns } \\
\text { weight (g) }\end{array}$ & $\begin{array}{c}188.71 \\
\pm 15.05^{\mathrm{a}, \mathrm{b}, \mathrm{c}, \mathrm{d}}\end{array}$ & $\begin{aligned} & 241.64 \\
\pm & 28.57^{\mathrm{a}, \mathrm{e}}\end{aligned}$ & $\begin{array}{c}230.61 \\
\pm 19.13^{\mathrm{b}, \mathrm{e}}\end{array}$ & $\begin{aligned} & 133.61 \\
\pm & 17.99^{\mathrm{c}, \mathrm{f}}\end{aligned}$ & $\begin{aligned} & 142.61 \\
\pm & 18.04^{\mathrm{d}, \mathrm{f}}\end{aligned}$ & $\begin{array}{l}0.03- \\
0.004\end{array}$ \\
\hline $\begin{array}{l}\text { Oviducts length } \\
\text { (cm) }\end{array}$ & $\begin{array}{c}38.66 \\
\pm 0.92^{\mathrm{a}, \mathrm{b}}\end{array}$ & $\begin{aligned} & 38.06 \\
\pm & 1.84^{\mathrm{a}, \mathrm{c}, \mathrm{d}}\end{aligned}$ & $\begin{aligned} & 37.76 \\
\pm & 1.20^{\mathrm{b}, \mathrm{c}}\end{aligned}$ & $\begin{array}{c}32.76 \\
\pm 1.18^{\mathrm{d}, \mathrm{e}}\end{array}$ & $\begin{array}{c}31.21 \\
\pm 1.17^{\mathrm{e}}\end{array}$ & $\begin{array}{l}0.04-< \\
0.0001\end{array}$ \\
\hline Oviducts weight (g) & $\begin{array}{c}2.79 \\
\pm 0.16\end{array}$ & $\begin{array}{r}3.08 \\
\pm 0.33\end{array}$ & $\begin{array}{c}3.05 \\
\pm 0.21\end{array}$ & $\begin{array}{c}2.60 \\
\pm 0.21\end{array}$ & $\begin{array}{c}2.60 \\
\pm 0.21\end{array}$ & ns \\
\hline $\begin{array}{l}\text { Ovary length, right } \\
(\mathrm{cm})\end{array}$ & $\begin{aligned} & 2.66 \\
\pm & 0.08^{\mathrm{a}, \mathrm{b}}\end{aligned}$ & $\begin{aligned} & 2.66 \\
\pm & 0.15^{\text {a,c }}\end{aligned}$ & $\begin{aligned} & 2.61 \\
\pm & 0.10^{\mathrm{b}, \mathrm{c}}\end{aligned}$ & $\begin{aligned} & 2.04 \\
& \pm 0.09^{d}\end{aligned}$ & $\begin{aligned} & 2.02 \\
\pm & 0.09^{d}\end{aligned}$ & $\begin{array}{l}0.01-< \\
0.0001\end{array}$ \\
\hline $\begin{array}{l}\text { Ovary length, left } \\
\text { (cm) }\end{array}$ & $\begin{aligned} & 2.62 \\
\pm & 0.09^{\mathrm{a}, \mathrm{b}, \mathrm{c}}\end{aligned}$ & $\begin{array}{c}2.59 \\
\pm 0.17^{\text {a,d,e,f }}\end{array}$ & $\begin{aligned} & 2.83 \\
\pm & 0.11^{\mathrm{b}, \mathrm{d}}\end{aligned}$ & $\begin{aligned} & 2.16 \\
\pm & 0.10^{\mathrm{e}, \mathrm{g}}\end{aligned}$ & $\begin{array}{c}2.25 \\
\pm 0.10^{\text {c,f,g }}\end{array}$ & $\begin{array}{l}0.02- \\
0.001\end{array}$ \\
\hline $\begin{array}{l}\text { Ovary weight, right } \\
\text { (g) }\end{array}$ & $\begin{aligned} & 4.35 \\
\pm & 0.37^{\mathrm{a}, \mathrm{b}}\end{aligned}$ & $\begin{array}{c}4.07 \\
\pm 0.70^{\mathrm{a}, \mathrm{c}, \mathrm{d}, \mathrm{e}}\end{array}$ & $\begin{array}{c}3.91 \\
\pm 0.47^{\text {b,c,f,g }}\end{array}$ & $\begin{aligned} & 2.45 \\
\pm & 0.43^{\mathrm{d}, \mathrm{f}, \mathrm{h}}\end{aligned}$ & $\begin{array}{c}2.25 \\
\pm 0.44^{\mathrm{e}, \mathrm{g}, \mathrm{h}}\end{array}$ & $\begin{array}{l}0.01- \\
0.006\end{array}$ \\
\hline $\begin{array}{l}\text { Ovary weight, left } \\
\text { (g) }\end{array}$ & $\begin{aligned} & 4.73 \\
\pm & 0.38^{\mathrm{a}, \mathrm{b}}\end{aligned}$ & $\begin{array}{c}3.97 \\
\pm 0.75^{\mathrm{a,c,d}, \mathrm{e}}\end{array}$ & $\begin{array}{c}4.51 \\
\pm 0.49^{\mathrm{b}, \mathrm{c}, \mathrm{f}, \mathrm{g}}\end{array}$ & $\begin{array}{c}2.73 \\
\pm 0.49^{\mathrm{d}, \mathrm{f}, \mathrm{h}}\end{array}$ & $\begin{array}{c}2.60 \\
\pm 0.51^{\mathrm{e}, \mathrm{g}, \mathrm{h}}\end{array}$ & $0.02-0.01$ \\
\hline $\begin{array}{l}\text { Ovary width, right } \\
(\mathrm{cm})\end{array}$ & $\begin{aligned} & 1.80 \\
\pm & 0.07^{\mathrm{a} b \mathrm{~b}}\end{aligned}$ & $\begin{aligned} & 1.83 \\
\pm & 0.13^{\text {a,c }}\end{aligned}$ & $\begin{aligned} & 1.79 \\
\pm & 0.09^{\mathrm{b}, \mathrm{c}}\end{aligned}$ & $\begin{array}{l}1.36 \\
\pm 0.08^{d}\end{array}$ & $\begin{array}{l}1.33 \\
\pm 0.08^{\mathrm{d}}\end{array}$ & $\begin{array}{l}0.04- \\
0.002\end{array}$ \\
\hline $\begin{array}{l}\text { Ovary width, left } \\
(\mathrm{cm})\end{array}$ & $\begin{aligned} & 1.88 \\
\pm & 0.08^{\mathrm{a}, \mathrm{b}}\end{aligned}$ & $\begin{array}{c}1.77 \\
\pm 0.14^{\mathrm{a}, \mathrm{c}, \mathrm{d}, \mathrm{e}}\end{array}$ & $\begin{aligned} & 1.93 \\
\pm & 0.10^{\mathrm{b}, \mathrm{c}}\end{aligned}$ & $\begin{array}{l}1.47 \\
\pm 0.09 \mathrm{~d}, \mathrm{f}\end{array}$ & $\begin{aligned} & 1.44 \\
\pm & 0.09^{\mathrm{e}, \mathrm{f}}\end{aligned}$ & $\begin{array}{l}0.02- \\
0.007\end{array}$ \\
\hline
\end{tabular}

\section{Discussion}

The present study shows strong associations between the $A R$ genotype and fat traits within the DUMI resource population. The results are consistent with the X-linked QTL for fatness traits previously reported in the pig (KNOTT et al., 1998; HARLIZIUS et al., 2000; ROHRER, 2000). As expected, the "M" allele from Berlin Miniature pig associates with high fat deposition. Like observed in other resource populations the alleles originated from Asian breeds (the Berlin Miniature Pig was breed from German Landrace, Saddleback and Vietnamese Potbelly Pig) increase back fat except those on chromosome 7. Moreover, dose dependent responses of the $\mathrm{M}$ allele on fatness were likely to be present in females but not statistically significant. A small number of homozygous MM may be a cause. Since the $A R$ is located on the $\mathrm{X}$ chromosome, on the other side, deviations from a random $\mathrm{X}$-inactivation in heterozygous females may be occurred and this can hide the allele effects (GARTLER and GOLDMAN, 2001; BROWN, 2001).

There are evidences suggesting that the $A R$ has direct and/or indirect effects on adipose tissue and hence fat depositions. It has been found that plasma testosterone level associated with back fat thickness and weight gain in line selected boars (ANDRESEN, 1976). The $A R$ is highly expressed in preadipocytes and mature 
adipocytes (DIEUDONNE et al., 1998). It has been shown that the $A R$ can directly determine the mesenchymal precursor cell into adipogenic lineage (in vitro) by acting at sites of myogenic and adipogenic differentiation pathways (SINGH et al., 2003). De PERGOLA (2000) reported that testosterone inhibits differentiation of adipocyte precursor cells, suppresses lipid uptake and lipoprotein lipase activity in adipocytes, and up-regulates the number of $\beta$-adrenergic receptors. Androgens may also affect fat deposition by inhibiting expression of peroxisomal proliferators-activated receptor $\gamma 2$ (PPAR $\gamma 2)$ and CCAAT/enhancer binding protein $\alpha(\mathrm{C} / \mathrm{EBP} \alpha)$ which are transcription factors regulating adipogenic differentiation. Moreover, the effects of the $A R$ on fat traits may be mediated through leptin pathway influencing feed intake and energy balance since testosterone can partly regulates leptin expression and secretion in adipocytes (WAUTERS et al., 2000).

QTL for reproduction traits, including traits related to litter size, reproductive organs measurements, puberty and gestation development have been assigned to several autosomes, however there is only one QTL for FSH plasma concentration on SSCX (ROHRER et al., 2001). Here we provide evidence for a QTL for uterus and ovary dimension on the $\mathrm{X}$ chromosome. The present results showed that the $A R$ genotypes affect uterus and ovary measurements, which are important components of reproductive traits in pigs (FORD et al., 2002). Not surprising that alleles from the Berlin Miniature pig associate with long horns of uterus and large ovary weight since genetics of Asian-related breeds is generally expected to have positive influence on prolificacy. Size of vagina and cervix are not affected by AR genotype or show inverse effect of Duroc or Miniature pig originated alleles, respectively. Variation in cervix and vagina size parallels variation in body size to a higher extent than size of uterus and ovary that are of higher functional importance with regard to fertility. Similar results have been observed in a study comparing uterine dimension of Large White and Meishan gilts at the same physiological stages (BAZER et al., 1988). Effects of breeds influencing length of uterine horns were reported in the study of pure breed Large White, Yorkshire and crossbred (ISLER et al., 2002).

The $A R$ could have diverse effects depending on stages of developments since it functions as nuclear transcription factors determining up- or down-regulation of genes in response to sex hormones. It has been shown that the female reproductive tract is under negative control of androgens (DREWS et al., 2002). On the other hand, the androgen receptor together with other nuclear receptors cooperatively plays important roles in functional phases of uterus. For example, estrogen administration positively regulates and restores the $A R$ mRNA expression in mouse uterus and vagina after ovarectomy (PELLETIER et al., 2004). The $A R$ is detected in nuclei of most cell types of pig uterus including epithelial, stroma, myometrial, and endothelial cells (CARDENAS and POPE, 2003). It presents mainly in glandular and luminal epithelia and remains unchanged throughout the estrous cycle and early pregnancy. Besides, the $A R$ expresses abundantly in granulosa cells of growing follicles and promotes follicle development (WEIL et al., 1998). These indicate that the $A R$ interacts with other genes at physiological levels to determine sex organ development and hence potentially influences various fertility related traits.

The present results show strong associations between the $A R$ genotypes and fatness traits, length of uterus horns and weight of ovary in pigs. Taking into account linkage 
disequilibrium that is expected in an intercross of divergent breeds the present results confirm the previously reported QTL for fatness traits on SSCX and for the first time provide evidence for a QTL affecting dimensions of uterus and ovary on the $\mathrm{X}$ chromosome in the pig. The study promotes AR as a positional functional candidate gene for traits related to obesity and reproduction. Our previous study showed a number of polymorphisms within the gene and SNPs in the promoter. These polymorphisms are likely to affect gene function and expression and consequently to influence biochemical pathways and physiology of associated traits. Further studies in vivo and in vitro - will elucidate and prove whether AR represents the QTL and the polymorphisms detected represent the genomic variations underlying the effects on phenotypic variation.

\section{Acknowledgements}

We thank PD Dr. Torsten Hardge for providing data of measurements of reproductive organs and ADir Dr. Ernst Tholen for his valuable advises for statistical analyses.

ANDRESEN, O.:

\section{References}

Concentrations of fat and plasma 5alpha-androstenone and plasma testosterone in boars selected for rate of body weight gain and thickness of back fat during growth, sexual maturation and after mating. $J$. Reprod. Fertil. 48 (1976), 51-59

BAZER, F.W.; THATCHER, W.W.; MARTINAT-BOTTE, F.; TERQUI, M.:

Sexual maturation and morphological development of the reproductive tract in large white and prolific Chinese Meishan pigs. J. Reprod. Fertil. 83 (1988), 723-728

BRINKMANN, A.O.:

Lessons to be learned from the androgen receptor. Eur. J. Dermatol. 11 (2001), 301-303

\section{BROWN, C.J.:}

Equality of the sexes: mammalian dosage compensation. Semin. Reprod. Med. 19 (2001), 125-132

CARDENAS, H.; POPE, W.F.:

Distribution and changes in amounts of the androgen receptor in the pig uterus during the estrous cycle, early pregnancy and after treatment with sex steroids. J. Endocrinol. 177 (2003), 461-469

De PERGOLA. G.:

The adipose tissue metabolism: role of testosterone and dehydroepiandrosterone. Int. J. Obes. Relat. Metab. Disord. Suppl. 2 (2000), 59-63

DIEUDONNE. M.N.; PECQUERY, R.; BOUMEDIENE, A.; LENEVEU, M.C.; GIUDICELLI, Y.: Androgen receptors in human preadipocytes and adipocytes: regional specificities and regulation by sex steroids. Am. J. Physiol. 274 (1998), 1645-1652

DREWS, U.; SULAK, O.; SCHENCK, P.A.:

Androgens and the development of the vagina. Biol. Reprod. 67 (2002), 1353-1359

FORD, S.P.; VONNAHME, K.A.; WILSON, M.E.:

Uterine capacity in the pig reflects a combination of uterine environment and conceptus genotype effects. J. Anim. Sci. 80 (2002), 66-73

GARTLER, S.M.; GOLDMAN, M.A.: Biology of the X chromosome. Curr. Opin. Pediatr. 13 (2001), 340-345

HARDGE, T.; KOEPKE, K.; REISSMANN, M.; WIMMERS, K.:

Maternal influences on litter size and growth in reciprocal crossed Miniature Pigs and Durocs. Arch. Tierz., Dummerstorf 42 (1999), 83-92

HARLIZIUS, B.; RATTINK, A.P.; KONING, De D.J.; FAIVRE, M.; JOOSTEN, R.G.; ARENDONK, van J.A.; GROENEN, M.A.:

The $\mathrm{X}$ chromosome harbors quantitative trait loci for backfat thickness and intramuscular fat content in pigs. Mamm. Genome 11 (2000), 800-802

ISLER, B.J.; IRVIN, K.M.; NEAL, S.M.; MOELLER, S.J.; DAVIS, M.E.:

Examination of the relationship between the estrogen receptor gene and reproductive traits in swine. J. Anim. Sci. 80 (2002), 2334-2339 
KNOTT, S.A.; MARKLUND, L.; HALEY, C.S.; ANDERSSON, K.; DAVIES, W.; ELLEGREN, H.; FREDHOLM, M.; HANSSON, I.; HOYHEIM, B.; LUNDSTROM, K.; MOLLER, M.; ANDERSSON, L.: Multiple marker mapping of quantitative trait loci in a cross between outbred wild boar and large white pigs. Genetics 149 (1998), 1069-1080

MALEK, M.; DEKKERS, J.C.; LEE, H.K.; BAAS, T.J.; ROTHSCHILD, M.F.:

A molecular genome scan analysis to identify chromosomal regions influencing economic traits in the pig. I. Growth and body composition. Mamm. Genome 12 (2001a), 630-636

MALEK, M.; DEKKERS, J.C.; LEE, H.K.; BAAS, T.J.; PRUSA, K.; HUFF-LONERGAN, E.; ROTHSCHILD, M.F.:

A molecular genome scan analysis to identify chromosomal regions influencing economic traits in the pig. II. Meat and muscle composition. Mamm. Genome 12 (2001b), 637-645

McPHAUL, M.J.; MARCELLI, M.; ZOPPI, S.; GRIFFIN, J.E.; WILSON, J.D.:

Genetic basis of endocrine disease. 4. The spectrum of mutations in the androgen receptor gene that causes androgen resistance. J. Clin. Endocrinol. Metab. 76 (1993), 17-23

NANTERMET, P.V.; XU, J.; YU, Y.; HODOR, P.; HOLDER, D.; ADAMSKI, S.; GENTILE, M.A.; KIMMEL,

D.B.; HARADA, S.; GERHOLD, D.; FREEDMAN, L.P.; RAY, W.J.: Identification of genetic pathways activated by the androgen receptor during the induction of proliferation in the ventral prostate gland. J. Biol. Chem. 279 (2004), 1310-1322

NELSON, P.S.; CLEGG, N.; ARNOLD, H.; FERGUSON, C.; BONHAM, M.; WHITE, J.; HOOD, L.; LIN, B.: The program of androgen-responsive genes in neoplastic prostate epithelium. Proc. Natl. Acad. Sci. U S A 99 (2002), 11890-11895

O'CONNELL, J.R.; WEEKS. D.E.: PedCheck: a program for identification of genotype incompatibilities in linkage analysis. Am. J. Hum. Genet. 63 (1998), 259-266

PELLETIER, G.:

Localization of androgen and estrogen receptors in rat and primate tissues. Histol. Histopathol. 15 (2000), 1261-1270

PELLETIER, G.; LUU-THE, V.; LI, S.; LABRIE, F.:

Localization and estrogenic regulation of androgen receptor mRNA expression in the mouse uterus and vagina. J. Endocrinol. 180 (2004), 77-85

RIS-STALPERS, C.; KUIPER, G.G.; FABER, P.W.; SCHWEIKERT, H.U.; ROOIJ, van H.C.; ZEGERS, N.D.;

HODGINS, M.B.; DEGENHART, H.J.; TRAPMAN, J.; BRINKMANN, A.O.:

Aberrant splicing of androgen receptor mRNA results in synthesis of a nonfunctional receptor protein in a patient with androgen insensitivity. Proc. Natl. Acad. Sci. U S A 87 (1990), 7866-7870

ROHRER, G.A.:

Identification of quantitative trait loci affecting birth characters and accumulation of backfat and weight in a Meishan-White Composite resource population. J. Anim. Sci. 78 (2000), 2547-2553

ROHRER, G.A.; KEELE. J.W.:

Identification of quantitative trait loci affecting carcass composition in swine: II. Muscling and wholesale product yield traits. J. Anim. Sci. 76 (1998), 2255-2262

ROHRER, G.A.; WISE. T.H.; LUNSTRA, D.D.; FORD, J.J.:

Identification of genomic regions controlling plasma FSH concentrations in Meishan-White Composite boars. Physiol. Genomics. 6 (2001), 145-151

SINGH, R.; ARTAZA, J.N.; TAYLOR, W.E.; GONZALEZ, C.A.; DAVID, N.F.; BHASIN, S.: Androgens stimulate myogenic differentiation and inhibit adipogenesis in C3H 10T1/2 pluripotent cells through an androgen receptor-mediated pathway. Endocrinology 144 (2003), 5081-5088

SLOMCZYNSKA, M.; DUDA, M.; SL, zak K.:

The expression of androgen receptor, cytochrome P450 aromatase and FSH receptor mRNA in the porcine ovary. Folia Histochem. Cytobiol. 39 (2001), 9-13

TRAKOOLJUL, N.; PONSUKSILI, S.; SCHELLANDER, K.; WIMMERS, K.:

A highly polymorphic repetitive polypyrimidine/polypurine (CCTTT)n sequence in the 5' untranslated sequence of the porcine androgen receptor gene. Anim. Genet. 31 (2000), 288-289

TRAKOOLJUL, N.; PONSUKSILI, S.; SCHELLANDER, K.; WIMMERS, K.: Polymorphisms of the porcine androgen receptor gene affecting its amino acid sequence and expression level. Biochim. Biophys. Acta 1678 (2004), 94-101

TRIPEPI, S.; CARELli, A.; PERROTTA, E.; BRUNELlI, E.; TAVOLARO, R.; FACCIOLO, R.M.; CANONACO, M.:

Morphological and functional variations of Leydig cells in testis of the domestic pig during the different biological stages of development. J. Exp. Zool. 287 (2000), 167-175

WAUTERS, M.; CONSIDINE, R.V.; GAAL, van L.F.:

Human leptin: from an adipocyte hormone to an endocrine mediator. Eur. J. Endocrinol. 143 (2000), 293-311 
WEIL, S.J.; VENDOLA, K.; ZHOU, J.; ADESANYA, O.O.; WANG, J.; OKAFOR, J.; BONDY, C.A.: Androgen receptor gene expression in the primate ovary: cellular localization, regulation, and functional correlations. J. Clin. Endocrinol. Metab. 83 (1998), 2479-2485

Received: 2005-06-22

Accepted: 2005-07-18

Author's addresses

PD Dr. KLAUS WIMMERS*

Research Institute for the Biology of Farm Animals (FBN), Research Unit Molecular Biology, Wilhelm-Stahl-Allee 2, 18196 DUMMERSTORF, GERMANY

PD Dr. SIRILUCK PONSUKSILI

Research Institute for the Biology of Farm Animals (FBN), Research Group Functional Genomics, Wilhelm-Stahl-Allee 2, 18196 DUMMERSTORF, GERMANY

Dr. NARES TRAKOOLJUL, Prof. Dr. KARL SCHELLANDER Institute of Animal Science, University of Bonn, Endenicher Allee 15, 53115 BONN, GERMANY

*correspondence to: Klaus Wimmers

E-mail: wimmers@fbn-dummerstorf.de 